\title{
SOME INEQUALITIES OF DIFFERENTIAL POLYNOMIALS II
}

\author{
JUNFENG XU, HONGXUN Yi AND ZHANLIANG ZHANG
}

Abstract. In this paper, we consider the value distribution of the differential polynomials $f^{2} f^{(k)}$ 1 where $k$ is a positive integer, and obtain some estimates only by the reduced counting function. Our result answers a question in (Some inequalities of differential polynomials, Mathematical Inequalities and Applications, 12, 1(2009), 99-113) completely.

Mathematics subject classification (2010): 30D35, 26D10. distribution.

Keywords and phrases: Meromorphic function; differential polynomials; Nevanlinna theory; value

\section{REFERENCES}

[1] W. Bergeiler AND A. EREmenko, On the singularities of the inverse to a meromorphic function of finite order, Rev. Mat. Iberoamericana., 11 (1995), 355-373.

[2] J. Clunie, On integral and meromorphic functions, J. London Math. Soc., 37 (1962), 17-27.

[3] F. Gackstatter, I. Laine, Zur Theorie der gewöhnlichen Differentialgleichungen im Komplexen, Ann. Polon. Math., 38 (1980), 259-287.

[4] W. K. Hayman, Meromorphic functions, Clarendon Press, Oxford, 1964.

[5] X. J. Huang And Y. X. Gu, On the value distribution of $f^{2} f^{(k)}$, J. Aust. Math. Soc., 78(2005), $17-26$.

[6] I. LAINE, Nevanlinna theory and complex differential equations, Walter de Gruyter, Berlin-New York, 1993.

[7] J. F. Xu, H. X. Yi And Z. L. Zhang, Some inequalities of differential polynomials, Mathematical Inequalities and Applications, 12, 1 (2009), 99-113.

[8] K. YAMANOI, The second main theorem for small functions and related problems, Acta Math., 192 (2004), 225-294.

[9] C. C. YANG AND H.X. Yi, Uniqueness Theory of Meromorphic Functions, New York, Dordrecht, Boston, London, 2003.

[10] L. YANG, Value distribution theory, Springer, Berlin, Heidelberg, New York, 1993.

[11] Q. D. ZHANG, A growth theorem for meromorphic functions, J. Chengdu Inst. Meteor., 20 (1992), $12-20$. 